\title{
Safety Grade Evaluation of Aqueduct Structure Based on Fuzzy Cloud Theory Analysis
}

\author{
Yu CHENG, Zhengzhong WANG, Cundong XU, Hui CHENG, Xinglin ZHU
}

\begin{abstract}
In view of the limitation of natural conversion between qualitative concept and quantitative value, the fuzzy analysis method cannot be employed to improve the multi-stage fuzzy evaluation method. Improvement of multilevel fuzzy evaluation method based on cloud theory was found, which could comprehensively consider the uncertainty of random combinations and the fuzziness of interaction between influencing factors, and the shortcomings of conventional fuzzy evaluation methods as well eliminated. The structure of 5\#U-aqueduct in Jingdian Irrigation District (Gansu, China) was chosen as the research object. Based on the multi-level fuzzy evaluation index system of structural safety, experts were invited to score the importance of factors according to the cloud theory scale criterion to establish a judgment matrix. Therefore, the evaluation set, weight and membership cloud model of evaluation system was constituted by means of bridge safety assessment criterion and cloud generator principle. Furthermore, comprehensive evaluation results of aqueduct service states were obtained by multistage fuzzy composite mapping method, and the actual position of structural safety grade was obtained by comparing the evaluation result with the comment layer cloud drop diagram. The results showed that using cloud model parameters (Ex, En and $\mathrm{He}$ ) to describe the relative importance of factors can better reflect the randomness and fuzziness of each other than the traditional single number, which was helpful to get the evaluation results accurately and objectively; The comprehensive evaluation results of the aqueduct structure obtained by calculation were $W(75.149,9.95,4.16)$. The simulated cloud droplet diagram was located between II and III classes, and tends to the III standard, which indicated that the overall security of the structure was sufferable. However, attention should be paid to the maintenance and repair of the detailed components. The consequences agreed with the evaluation results of the experts, which indicates that the improved evaluation method has good practicability as well as can be popularized and applied.
\end{abstract}

Keywords: aqueduct; cloud generator; cloud theory; comment set; membership degree; multistage fuzzy evaluation; safety condition; weight

\section{INTRODUCTION}

In long-distance water transportation projects, aqueducts are commonly used cross-ditch buildings. The service state and safety of these aqueduct structures have a great impact on the safe operation of the entire water transfer project. Among the 402 large-scale irrigation districts built in the 1970s and 1980s in China, the aqueduct structure was a widely adopted and indispensable type of water delivery structure [1]. These buildings have been in service for a long time under the influence of complex environmental media. Most of the structural damage occurred in different degrees. In order to ensure the safe and reliable operation of these projects, the Ministry of Water Resources began to carry out the continuous construction and renovation of large-scale irrigation districts in 2001. During the renovation, the classification of the damage degree of important structures such as aqueducts and tunnels has gradually attracted people's attention [2,3]. Detecting the major factors affecting structural safety and its coupling mechanism, avoiding the traditional safety assessment mainly based on human experience judgment and the subjective randomness of evaluation results, is qualitatively also more specific for repair and reinforcement of the structure. Timely evaluation of the service state of the structure should be made to determine the degree of damage and safety. Many researches have been done by domestic and foreign scholars on these issues. Sun Bo \& Xiao Rucheng [4] introduced fuzzy mathematics into the analytic hierarchy process and combined the advantages of the two to evaluate the safety level of bridge engineering. However, this method fails to comprehensively consider the randomness and uncertainty between the influence factor data. Zhan Jiawang, Yan Yuzhi et al. [5] used the model correction technique and optimization algorithm to evaluate the health of the lower structure of the simply supported beam bridge, but the solution results of this method depended greatly on the initial value and only the local optimal solution could be obtained. Fenocchi A. \& Natale L. [6] used a numerical physical model to evaluate the degree of erosion of the structure and determined its damage level. However, the method is aimed at a single object and has a narrow application range. Hu Junliang, Zhong Jiwei et al. [7] determined the reliability index of the artificial neural network improved response surface method to determine the security status level of the structure and proposed a solution, but this method cannot deal with fuzzy information, and the utilization rate of expert judgment is low. These research results provide a reference method for the structural safety level evaluation of hydraulic structures, but the damage degree of the whole building is deduced from the damage degree of the detailed components of the structure itself. At the same time, the fuzziness and uncertainty of multi-factor coupling effects affecting structural safety are considered less. The fuzzy interaction process for structural composition forms, design criteria, influencing factors and durability defects cannot be quantitatively described and judged. Therefore, in order to comprehensively consider the multi-level influencing factors and their function systems that affect the safety of buildings, and to combine the uncertainties such as ambiguity, randomness and volatility between these element data, it is necessary to study the construction and evaluation methods of multi-level coupling systems that affect structural safety.

This paper selects the $5^{\#} U$-type aqueduct structure of the main canal in Jingdian Irrigation District of Gansu Province, China, as the research object. It is carried out in five aspects, the multi-level decomposition of structural form, seismic performance, bearing capacity, service status and durability defects. Considering the fuzzy discreteness of the interaction between the components of the building, and considering the indirect effects of the maintenance measures during the service process, it also takes into account the randomness of the combined effects of the working conditions load and the material durability damage. The cloud theory is introduced into the multi-level 
fuzzy evaluation model. The cloud model parameters that characterize the uncertainty are used to replace the single evaluation data in the fuzzy evaluation. The improved evaluation method is used to classify the service status of the aqueduct and determine the damage degree and the maintenance program. It provides a reference method for damage assessment of similar structures in the same environment. Since the water conservancy industry has not issued normative evaluation standards and assessment criteria for aqueduct structures, considering that the water conveyance aqueduct is similar in structure and function to road and bridge structures, this paper refers to "the standard of the safety assessment method of bridge engineering and the assessment of highway bridge technical status. (JTG/T H21-2011)" to evaluate the service safety of the aqueduct structure.

\section{MULTI-LEVEL FUZZY EVALUATION MODEL OF AQUEDUCT STRUCTURE}

\subsection{Multi-Level Fuzzy Comprehensive Evaluation Model}

Pro. Wang Peizhuang proposed a fuzzy comprehensive evaluation model in 1980. After many scholars' improvements and updates in recent years, the evaluation method has gradually evolved from a traditional singlelevel comprehensive evaluation to a multi-level fuzzy comprehensive evaluation method [8]. The current multilevel fuzzy comprehensive evaluation method integrates the fuzzy evaluation method and the analytic hierarchy process. The solution process first decomposes the system to be evaluated into a multi-level hierarchical structure, and then determines the weight of a single index according to the analytic hierarchy process, and then uses fuzzy set theory to determine the fuzzy matrix of the relationship between the levels. Using this matrix to carry out fuzzy evaluation of each level in the system, finally gives the evaluation results of the whole system $[9,10]$.

Fuzzy set is a comprehensive evaluation method based on fuzzy mathematics. Its basic idea is to recognize the fuzziness existing in the process of development of things, and to think that the transition of the object studied in the assembly is gradually realized, not the abrupt phenomenon. In this way, the concept of absolute belonging is transformed into a concept of relative belonging. In the process of evaluation, whether the evaluation object belongs to a set is transformed into the problem of the membership degree of the evaluation object to a set. Moreover, the comprehensive evaluation is to grasp the target by grasping the law of its quantity change in the process of comparing the target, and to grasp the target from the fuzziness reflected by the interaction of a large number of single factors on the whole, so as to make a comprehensive description and evaluation of the goal. In this paper, a comprehensive evaluation of the safety of the aqueduct structure is carried out, whose durability decay process is influenced by the multi-level factors, which is characterized by the typical fuzziness and the uncertainty. The attenuation process is fuzzy and complex and the safety level of the final structure cannot be obtained through a single index. Therefore, in order to evaluate this chaotic process more comprehensively and objectively, the comprehensive evaluation model of multi-level fuzzy theory is adopted that is to say, the whole structure is divided one by one. The safety level of the structure is deduced by checking the usage status of each component.
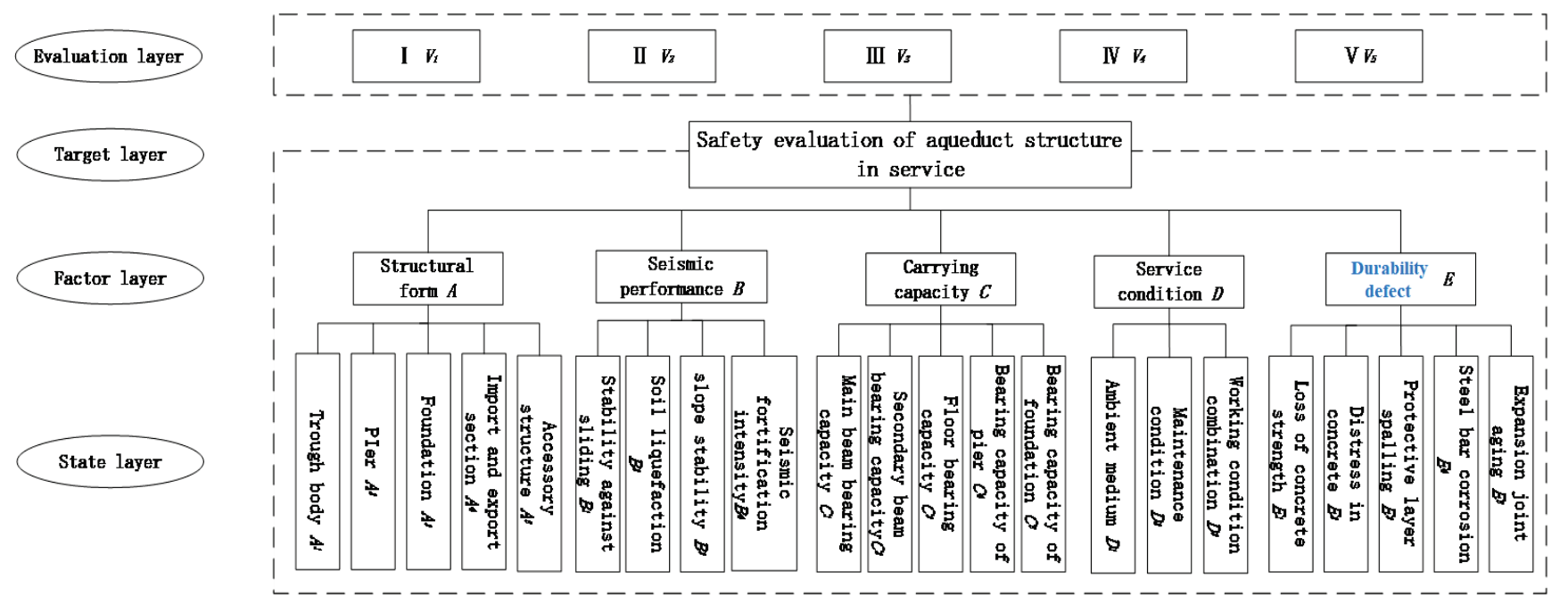

Figure 1 Multistage fuzzy evaluation index system of aqueduct safety performance

The aqueduct is also called water transfer bridge, and it is generally considered that the aqueduct belongs to a structure of the bridge, so in accordance with the Standard for Evaluation of the Project Quality of the Capital Engineering Unit of the Water and Hydropower Project (DL/T 5113.7-2015) and the Technical Conditions of Highway Bridge (JTG/T H21-2011), all kinds of factors that affect the safety and durability of the whole structure were comprehensively considered, and taken into consideration. Following the principles of objectivity, data availability, holism and popularity, the various indexes for evaluating aqueduct structure are selected, and a multilevel fuzzy evaluation system for aqueduct structure during service is established, as shown in Fig. 1.

The evaluation system consists of the evaluation layer, the target layer, the factor layer and the state layer. The evaluation layer (I, II, III, IV and V) indicates the service status of the aqueduct. The service completion degree of the aqueduct was evaluated and classified to provide the decision-making basis for the technical personnel's maintenance and renovation. The target layer was the service safety evaluation of the ultimate goal-aqueduct 
structure of the construction system $(U)$, and the factor layer was a large-classification result for each factor which influences the safety of the aqueduct, and comprises the structural form $(A)$, the anti-seismic performance $(B)$, the bearing capacity $(C)$, the service condition $(D)$ and the durability defect $(E)$. The state layer represents further interpretation and classification of the factor layer, for example, structural form $(A)$ includes trough body $\left(A_{1}\right)$, pier body $\left(A_{2}\right)$, foundation $\left(A_{3}\right)$, entrance and exit segment $\left(A_{4}\right)$, and subsidiary structure $\left(A_{5}\right)$. By this analogy, the residual constituent factors of the factor layer are reintegrated and classified.

\section{MULTI-LEVEL FUZZY EVALUATION MODEL IMPROVED BY THE CLOUD THEORY}

When the fuzzy evaluation model method is used to evaluate the safety level of the aqueduct, the fuzzy chaos of the complex system is considered, but the randomness and discreteness of the system are not discussed. And cloud theory can well describe the randomness and uncertainty of various factors in the system. Through the cloud theory, the relationship between qualitative concepts and quantitative values can be established and transformed, and the inherent relationship between randomness and chaos in the research system is revealed. Therefore, when the comprehensive fuzzy evaluation of the performance of the aqueduct structure during service is carried out, the uncertainty cloud theory is introduced into the multi-level fuzzy evaluation model to improve it.

\subsection{Basic Ideas of Cloud Theory}

A cloud model is a model that converts quantitative values and stereotyped concepts based on probability and statistics and fuzzy set theory. Each cloud drop in the model is characterized by the unique expectation $(E x)$, of this point. It is characterized by entropy $(E n)$ and super entropy $(H e)$, that is, $C(E x, E n, H e)[11,12]$. After introducing the cloud theory into the fuzzy evaluation system, the expectation (Ex) represents the cloud drop center position, which represents the weight of each level factor in the fuzzy evaluation system and the central value of the membership degree. Entropy (En) describes the ambiguity and randomness of cloud droplets, representing the ambiguity and dispersion of cloud droplet distribution, reflecting the uncertainty of the values of each layer factor in the system. Super entropy $(\mathrm{He})$ is the entropy of entropy, which is used to describe the degree of cloud droplet cohesion, which represents the degree of uncertainty of entropy, and reflects the deviation of the value of each layer factor from the central value in the system [13].

Normal cloud model is the most basic cloud model, which is universal and can reflect the fuzziness and uncertainty in the concept of things or human knowledge in the objective world. By completely integrating the two models, it forms a mapping between qualitative and quantitative. This paper provides a new method for the study of uncertainty problems [14]. Considering the scope of application of the evaluation method, the normal cloud model is used for the analysis.

Realization of conversion between qualitative concept and quantitative numerical value in Cloud Theory depends on Cloud Generator [15], which is divided into a forward cloud generator and a reverse cloud generator according to the direction of conversion, Among them, the forward cloud generator converts the qualitative into quantitative, that is, the cloud model parameters $(\mathrm{Ex}, \mathrm{En}, \mathrm{He})$ generate the droplet Drop $\left(x_{i}, y_{i}\right)$ [16]. The reverse cloud generator calculates the process. The reverse cloud generator calculation process, on the other hand, converts the cloud drop Drop $\left(x_{i}, y_{i}\right)$ into cloud model parameters $(E x, E n, H e)$, as shown in Fig. 2.

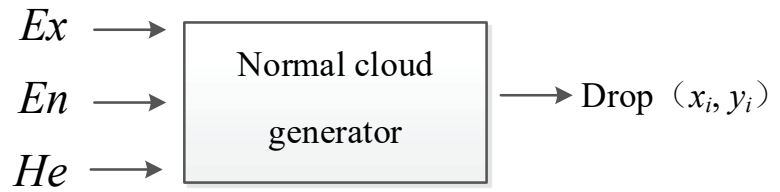

(a)

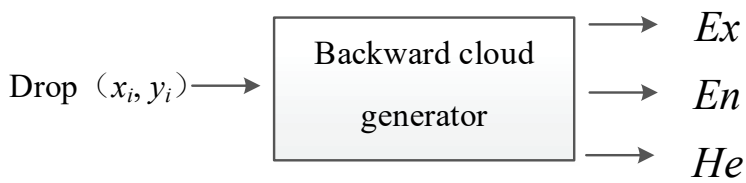

(b)

Figure 2 Cloud Generator: a) forward cloud generator; b) reverse cloud generator

\subsection{Induced Factor Weight Cloud Model}

The Analytic Hierarchy Process (AHP) requires an expert to select a natural number between 1 and 9 according to the Satty scale to indicate the relative importance between the two factors [17]. However, this scoring method cannot ignore the subjectivity of independent thinking of experts, and the fuzzy randomness of the relationship between factors cannot be displayed. Therefore, in the analysis of the relative importance of the detailed components of the service aqueduct, the use of cloud models to improve the traditional scale can achieve the objectivity and ambiguity of the evaluation process. The traditional scale criteria based on cloud model improvement are shown in Tab. 1.

Table 1 Component evaluation cloud model scale criterion for service aqueduct

\begin{tabular}{|c|c|c|}
\hline \multicolumn{2}{|c|}{$\begin{array}{l}\text { The importance of pairwise } \\
\text { comparisons }\end{array}$} & $\begin{array}{l}\text { Scale cloud model } \\
C(E x, E n, H e)\end{array}$ \\
\hline \multirow{4}{*}{$\begin{array}{l}S_{i} \text { is more important than } \\
S_{j}\end{array}$} & Absolutely & $C_{4}(9,0.33,0.01)$ \\
\hline & Strongly & $C_{3}(7,0.33,0.01)$ \\
\hline & Obviously & $C_{2}(5,0.33,0.01)$ \\
\hline & Slightly & $C_{1}(3,0.33,0.01)$ \\
\hline \multicolumn{2}{|c|}{$S_{i}$ as important as $S_{j}$} & $C_{0}(1,0,0)$ \\
\hline \multirow{4}{*}{$\begin{array}{c}S_{j} \text { is more important than } \\
S_{i}\end{array}$} & Slightly & $C_{5}(1 / 3, \quad 0.33 / 9, \quad 0.01 / 9)$ \\
\hline & Obviously & $C_{6}(1 / 5, \quad 0.33 / 25,0.01 / 25)$ \\
\hline & Strongly & $C_{7}(1 / 7, \quad 0.33 / 49, \quad 0.01 / 49)$ \\
\hline & Absolutely & $C_{8}(1 / 9, \quad 0.33 / 81, \quad 0.01 / 81)$ \\
\hline
\end{tabular}

The method of obtaining membership cloud model is based on the principle and calculation method of reverse cloud generator, which reflects the fuzziness and randomness of all kinds of components that represent the safety grade of aqueduct structure. The corresponding relationship between quantitative and qualitative factors can be further verified, and the membership degree of different factors at different levels to the target level factors can be made clear [18].

The evaluation set of determining aqueduct safety 
evaluation system was to satisfy the quantitative conversion and analysis of aqueduct durability, so the forward cloud generator was used to define the cloud model parameters $W_{i}^{q}\left(E x_{i}^{q}, E n_{i}^{q}, H e_{i}^{q}\right)$. Then the digital set of the evaluation layer in the evaluation model was obtained and a more intuitive image distribution can be obtained by simulation with MATLAB. Therefore, according to the multi-level fuzzy evaluation index system of aqueduct safety, on the basis of layer-by-layer classification, by determining the membership degree and weight of each node or level, the multi-level fuzzy composite mapping was adopted. The safety grade of aqueduct under fuzzy condition can be evaluated comprehensively.

\section{CASE ANALYSIS}

\subsection{Overview of Aqueduct Structure Engineering}

Gansu Jingtai chuan Electric Power Irrigation District (hereinafter referred to as "Jingdian Irrigation District") is a large (II) type water pumping irrigation project, in which the canal aqueduct accounts for $38.3 \%$ of the total number of buildings in these two projects [19]. It is an important cross-ditch building for water delivery in irrigation districts.
However, in the past 50 years of operation, due to the complex environmental media influence and long-term water service and other reasons, all kinds of detailed components of the aqueduct were damaged, such as foundation deformation, crack development, water stop failure, surface concrete spalling, etc. The damage of these components causes irreversible damage to the overall durability of the structure to varying degrees, which makes the actual service life of the aqueduct structure have a large gap with the expected target. When the irrigation district management department conducts routine maintenance on various types of hydraulic structures, due to the lack of scientific evaluation of the structural service status and the deterministic analysis of the impact factors, the phenomenon that the engineering maintenance is subjectively judged by experience and technical personnel is widespread. Therefore, the total dry $5^{\sharp} U$-type aqueduct structure of "Jingdian Irrigation District" was selected as the evaluation object, and the overall service state was comprehensively evaluated according to the weight and damage degree of different components of the aqueduct structure. The structure diagram of the total dry $5^{\sharp} U$-type aqueduct in Jingdian Irrigation District is shown in Fig. 3.
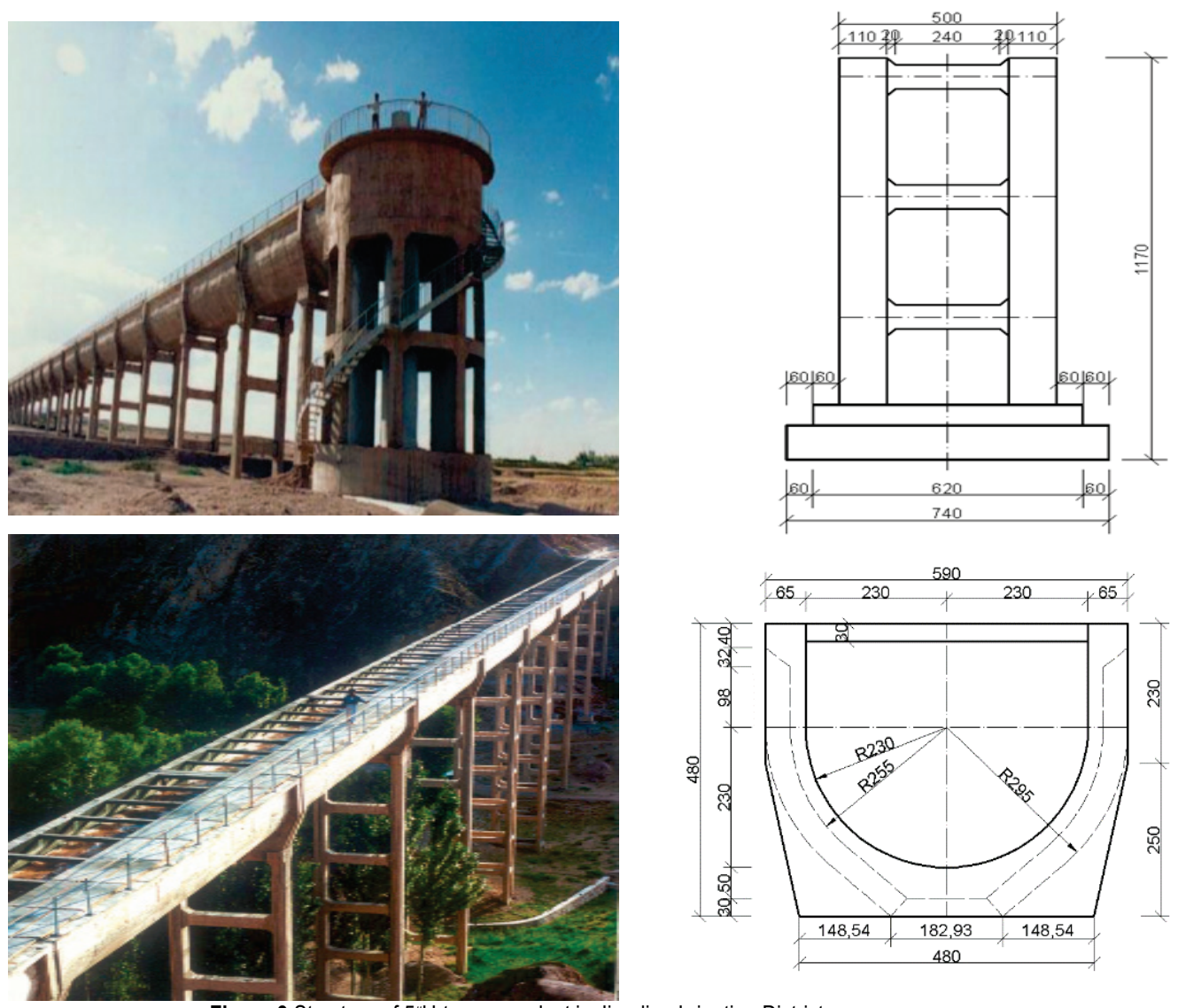

Figure 3 Structure of $5^{\# U-t y p e ~ a q u e d u c t ~ i n ~ J i n g d i a n ~ I r r i g a t i o n ~ D i s t r i c t ~}$

\subsection{Assessment of Service Status of Aqueduct Structure 4.2.1 Review Cloud Model of Aqueduct Structure}

According to the Standards for the Evaluation of Technical Conditions of Highway Bridges (JTG/T H212011), the assessment level of the aqueduct structure is evaluated by the bridge evaluation grade, in order to achieve the goal of guiding the aqueduct maintenance and maintenance strategy. The specific manifestation is shown in Tab. 2. In the specification, the degree of damage of the structure is divided into five levels. From the Eqs. (1) to (3), the cloud model characteristic parameters corresponding to each level are calculated respectively. Among them, I and V $E x$, respectively, take 100 and 0 [20], the specific results are shown in Tab. 2. 


$$
\begin{aligned}
& E x_{i}^{\mathrm{V}}=\frac{\left(x_{i}^{1}+x_{i}^{2}\right)}{2} \\
& E n_{i}^{\mathrm{V}}=\sqrt{\frac{\left(x_{i}^{1}+x_{i}^{2}\right)^{2}}{8 \cdot \ln 2}}
\end{aligned}
$$

$H e=k$
In the formula, $x_{i}^{1}$ and $x_{i}^{2}$ represent the upper and lower boundary values of the aqueduct safety level (I, II, III, IV and V) in the evaluation set respectively, and the damage degree is often used to determine the grade. Using the forward cloud generator, the target layer of comprehensive evaluation of the service degree of the aqueduct structure is simulated and displayed by using the Matlab software as in Fig. 4.

\begin{tabular}{|c|c|c|c|c|c|c|}
\hline \multirow{2}{*}{ Evaluation level } & \multicolumn{3}{|c|}{ Evaluation conclusions and countermeasures } & \multicolumn{3}{|c|}{ Cloud model parameters } \\
\hline & overall ratings & Meaning & Countermeasures & $E x$ & $E n$ & $\mathrm{He}$ \\
\hline $\mathrm{I}$ & $95 \leq U<100$ & No damage & Daily maintenance & 100 & 2.12 & 0.031 \\
\hline II & $80 \leq U<95$ & Minor damage & Minor repair & 87.5 & 6.37 & 0.098 \\
\hline III & $60 \leq U<80$ & Moderate damage & Moderate repair & 70.0 & 8.49 & 0.357 \\
\hline IV & $40 \leq U<60$ & Serious damage & Overhaul or reinforcement & 50.0 & 8.49 & 0.643 \\
\hline $\mathrm{V}$ & $U<40$ & Total damage & Reconstruction & 0 & 16.99 & 0.883 \\
\hline
\end{tabular}

Table 2 Aqueduct structure safety evaluation level and cloud model parameter value

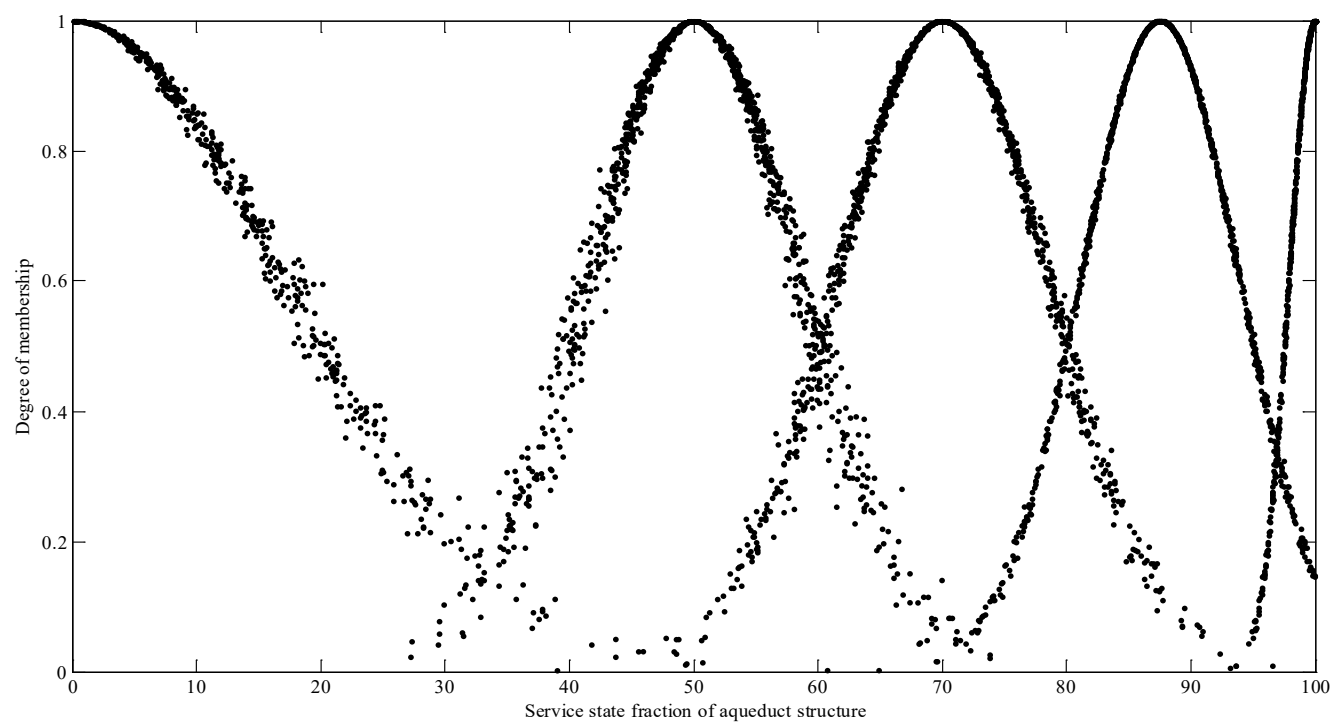

Figure 4 Cloud model of aqueduct security state evaluation

\subsubsection{Weight Cloud Model of Aqueduct Structure}

According to the existing engineering specifications, based on consulting the Jingdiang Irrigation District project operation management report and the relevant structural design materials, 9 experts including industry experts, engineering designers and management personnel were employed. According to the cloud model scale criterion constructed in Tab. 1, the importance degree of various factors affecting the service safety of the aqueduct structure was judged in pairs. Three judgment matrices based on the improved scale criterion were constructed respectively. The three judgment matrices were averaged and then calculated by Eqs. (4) to (6). The weight cloud model $W_{i}^{q}\left(E x_{i}^{q}, E n_{i}^{q}, H e_{i}^{q}\right)$ of available factors was obtained.

$$
E x_{i}^{0}=\frac{\left(\prod_{j=1}^{n} E x_{i j}\right)^{\frac{1}{n}}}{\sum_{i=1}^{n}\left(\prod_{j=1}^{n} E x_{i j}\right)^{\frac{1}{n}}}
$$

$$
\begin{aligned}
E n_{i}^{0}= & \frac{\left(\prod_{j=1}^{n} E x_{i j} \sqrt{\sum_{j=1}^{n}\left(\frac{E n_{i j}}{E x_{i j}}\right)^{2}}\right)^{\frac{1}{n}}}{\sum_{i=1}^{n}\left(\prod_{j=1}^{n} E x_{i j} \sqrt{\sum_{j=1}^{n}\left(\frac{E n_{i j}}{E x_{i j}}\right)^{2}}\right)^{\frac{1}{n}}} \\
& \sum_{i=1}^{n}\left(\prod_{j=1}^{n} E x_{i j}^{n} \sqrt{\sum_{j=1}^{n}\left(\frac{E e_{i j}}{E x_{i j}}\right)^{2}}\right)^{\frac{1}{n}}
\end{aligned}
$$

Then, in order to further verify the accuracy of expert scoring method, the Delphi method is used to verify the AHP, which is introduced into cloud theory, with the factor layer as an example. Because of the different forms of expression of the two results, the expected value Ex which reflects the factor weight in the method used in this paper 
is compared with the weight coefficient in the Delphi method. The comparison results are shown in Tab. 3 .

By comparing the results of the first row with the second row in Tab. 3, it can be found that the weight values obtained by the two methods were basically the same, the error was small, and the order of weights from large to small was also consistent. At the same time, combined with the specific values of $W_{i}^{q}\left(E x_{i}^{q}, E n_{i}^{q}, H e_{i}^{q}\right)$ in Tab. 3, the discreteness and fuzziness of the factors themselves are well guaranteed. It can be concluded that the AHP based on cloud theory can be used to analyze the weight of each index with higher reliability and less error. Therefore, continue to follow the Eqs. (4) to (6) for the cloud model parameters of the state layer factor as shown in Tab. 4.

Table 3 Aqueduct structure factor layer weight cloud mode

\begin{tabular}{|c|c|c|c|c|c|}
\hline Factor layer factor & Construction form $A$ & Seismic performance $B$ & Carrying capacity $C$ & Operation condition $D$ & Durability defect $E$ \\
\hline Delphi & 0.311 & 0.155 & 0.174 & 0.098 \\
\hline$E x$ & 0.264 & 0.159 & 0.189 & 0.262 \\
\hline$E n$ & 0.229 & 0.152 & 0.165 & 0.213 \\
\hline$H e$ & 0.211 & 0.142 & 0.154 & 0.124 & 0.124 \\
\hline
\end{tabular}

Table 4 Aqueduct structure state layer weight cloud model

\begin{tabular}{|c|c|c|c|c|c|c|c|c|c|c|c|c|}
\hline Factor layer factor & $A_{1}$ & $A_{2}$ & $A_{3}$ & $A_{4}$ & $A_{5}$ & $B_{1}$ & $B_{2}$ & $B_{3}$ & $B_{4}$ & $C_{1}$ & $C_{2}$ \\
\hline$E x$ & 0.057 & 0.054 & 0.051 & 0.055 & 0.047 & 0.047 & 0.025 & 0.044 & 0.045 & 0.052 & 0.036 \\
\hline$E n$ & 0.051 & 0.049 & 0.044 & 0.051 & 0.044 & 0.041 & 0.022 & 0.041 & 0.044 & 0.048 & 0.035 \\
\hline$H e$ & 0.050 & 0.049 & 0.044 & 0.048 & 0.046 & 0.42 & 0.020 & 0.041 & 0.042 & 0.048 & 0.031 \\
\hline Factor layer factor & $C_{3}$ & $C_{4}$ & $C_{5}$ & $D_{1}$ & $D_{2}$ & $D_{3}$ & $E_{1}$ & $E_{2}$ & $E_{3}$ & $E_{4}$ & $E_{5}$ \\
\hline$E x$ & 0.048 & 0.027 & 0.026 & 0.061 & 0.053 & 0.021 & 0.049 & 0.054 & 0.049 & 0.050 & 0.029 \\
\hline$E n$ & 0.044 & 0.027 & 0.023 & 0.056 & 0.049 & 0.023 & 0.045 & 0.050 & 0.045 & 0.045 & 0.029 \\
\hline$H e$ & 0.045 & 0.024 & 0.021 & 0.053 & 0.049 & 0.021 & 0.042 & 0.049 & 0.049 & 0.045 & 0.028 \\
\hline
\end{tabular}

\subsubsection{Membership Cloud Model of Aqueduct Structure}

In view of the 5 factor layer factors and 21 state layer factors which represent the safety of aqueduct structure in the Fig. 1, the expert score results were taken as the data basis, and the data were sorted out and analyzed, then based on the basic principle of the reverse cloud generator, the safety of aqueduct structure was analyzed. According to Eqs. (7) to (9), the membership function value of every factor of aqueduct structure in service under each state was established.

For a certain evaluation unit, the membership cloud model $W_{i}^{U}\left(E x_{U i}, E n_{U i}, H e_{U i}\right)$ of target layer and factor layer factor was extracted from the completed membership function, as shown in Tab. 5 and Tab. 6.

$$
\begin{aligned}
& E x=\frac{1}{n} \times \sum_{i=1}^{n} x_{i} \\
& E n=\sqrt{\frac{\pi}{2} \times \frac{1}{n} \times \sum_{i=1}^{n}\left|x_{i}-E x\right|} \\
& E e=\sqrt{\frac{1}{n-1} \times \sum_{i=1}^{n}\left(x_{i}-\bar{x}\right)^{2}-E n^{2}}
\end{aligned}
$$

Table 5 Aqueduct structure factor layer weight cloud model

\begin{tabular}{|c|c|c|c|c|c|}
\hline Factor layer factor & Operation management $D$ & Engineering quality $A$ & Seismic performance $B$ & Carrying capacity $C$ & Durability defect $E$ \\
\hline$E x$ & 82.31 & 78.65 & 82.13 & 76.19 \\
\hline$E n$ & 16.45 & 10.63 & 13.98 & 73.65 & 8.19 \\
\hline$H e$ & 8.33 & 5.89 & 6.66 & 3.99 & \\
\hline
\end{tabular}

Table 6 Aqueduct structure state layer weight cloud model

\begin{tabular}{|c|c|c|c|c|c|c|c|c|c|c|c|}
\hline Factor layer factor & $A_{1}$ & $A_{2}$ & $A_{3}$ & $A_{4}$ & $A_{5}$ & $B_{1}$ & $B_{2}$ & $B_{3}$ & $B_{4}$ & $C_{1}$ & $C_{2}$ \\
\hline$E x$ & 77.34 & 81.42 & 81.49 & 78.12 & 74.88 & 81.35 & 83.47 & 82.62 & 81.08 & 73.97 & 75.63 \\
\hline$E n$ & 73.51 & 79.56 & 78.94 & 77.52 & 71.66 & 79.01 & 80.96 & 80.02 & 78.56 & 70.29 & 71.99 \\
\hline$H e$ & 71.86 & 79.23 & 78.88 & 77.21 & 71.35 & 79.21 & 80.33 & 79.68 & 78.56 & 70.25 & 71.82 \\
\hline Factor layer factor & $C_{3}$ & $C_{4}$ & $C_{5}$ & $D_{1}$ & $D_{2}$ & $D_{3}$ & $E_{1}$ & $E_{2}$ & $E_{3}$ & $E_{4}$ & $E_{5}$ \\
\hline$E x$ & 73.17 & 78.22 & 79.96 & 79.89 & 84.56 & 82.48 & 75.84 & 70.23 & 72.44 & 76.85 & 72.89 \\
\hline$E n$ & 72.65 & 76.11 & 74.26 & 76.23 & 81.75 & 79.35 & 72.51 & 67.76 & 69.38 & 73.61 & 69.49 \\
\hline$H e$ & 72.65 & 76.02 & 74.26 & 75.89 & 82.01 & 79.11 & 72.64 & 67.76 & 69.25 & 73.33 & 69.36 \\
\hline
\end{tabular}

\subsubsection{Comprehensive Evaluation}

According to the weight cloud model and membership cloud model in Tab. 3 to Tab. 6, the safety level of aqueduct under fuzzy conditions is evaluated by using multi-level fuzzy composition mapping. The formula of the composition algorithm [22] is as follows:

$\boldsymbol{W}=\boldsymbol{X} \otimes Y$

$\boldsymbol{W}$ is the comprehensive evaluation matrix of the evaluation system, $\boldsymbol{X}$ is the weight vector of the aqueduct components, and $Y$ is the membership degree of the lowlevel elements to the high-level elements.

On the basis of Eq. (10), according to the principle of multi-stage fuzzy composition, the combination operation is carried out. That is to say, the comprehensive evaluation of the service state of the $5^{\#} \mathrm{U}$-aqueduct structure in Jingdian Irrigation District is based on the cloud model parameter $W^{\mathrm{V}}\left(E x^{\mathrm{V}}, E n^{\mathrm{V}}, H e^{\mathrm{V}}\right)=W^{\mathrm{V}}(75.149,9.95,4.16)$. Using the simulation function of Matlab, the parameters of the model are compared with the evaluation set $\left(V_{1}, V_{2}, V_{3}\right.$, $\left.V_{4}, V_{5}\right)$. The results are shown in Fig. 5.

It can be seen from Fig. 5 that the evaluation cloud 
droplets (red) that characterize the service state of the $5^{\#} \mathrm{U}$ type aqueduct structure were concentrated, and the reliability of the $E n$ representing the degree of ambiguity and the degree of randomness representing the degree of randomness were better, and the comprehensive evaluation results were more credible. The distribution of red cloud droplets was in the transition zone of $V_{2}$ and $V_{3}$ of the review set and had a tendency to develop toward $V_{3}$, which was consistent with the evaluation conclusions of the expert site survey. The results showed that the aqueduct structure was about to appear with medium defects that do not affect normal use, and the main component materials such as the tank body and the pier body tend to develop moderately damaged and begin to develop slow and mild functional diseases.

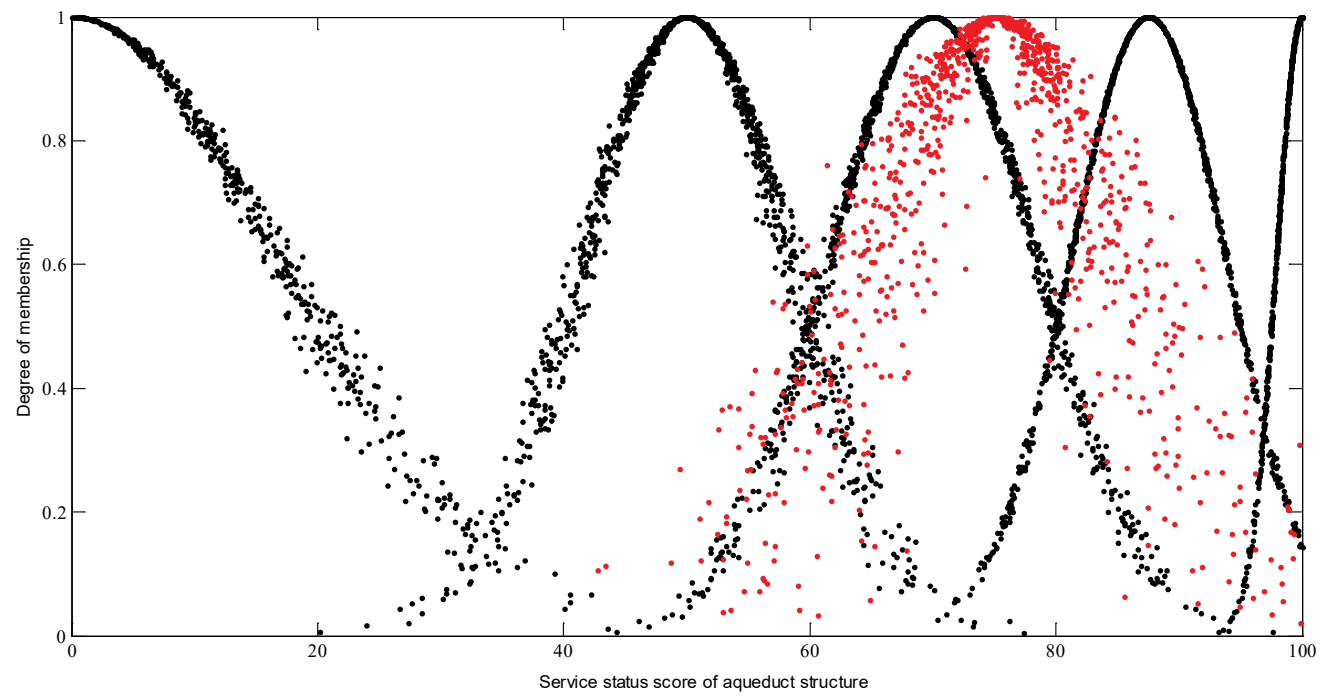

Figure 5 Comprehensive evaluation results of $5 \# \cup$ type aqueduct structure

At the same time, it should be noted that secondary parts such as expansion joints will soon reach a serious level of defects, and if further deteriorated, it would greatly affect the normal service of the main components. Therefore, the relevant responsible personnel and maintenance personnel of Jingdian Irrigation District need to probe the damage status of the secondary components of the aqueduct, and timely repair them to avoid radiation damage to the main components that would affect the overall water delivery function of the aqueduct. The performance indicators of the main components of the aqueduct should be monitored in real time, and timely response measures should be taken for areas with moderate or functional diseases to slow down the defect rate of the main components.

\section{DISCUSSION}

In order to further verify the superiority of the fuzzy evaluation method based on cloud theory, the service status of $5^{\#} \mathrm{U}$-aqueduct in Jingdian irrigation area is evaluated by using conventional fuzzy evaluation method based on the existing data. The comprehensive evaluation matrix $\boldsymbol{W}=$ $(82.3,76.2,79.8,78.4,75.1)$ was obtained. According to the principle of maximum membership, considering the factors causing most damage in this assessment, it is deduced that the service safety grade of the target aqueduct structure is III.

But compared with the actual monitoring results, the evaluation results obtained by the method proposed in this paper are more consistent with the actual estimation results, that is, the aqueduct safety level is between II and III. Compared with the results obtained by the two methods, the conventional fuzzy evaluation method cannot take into account the random effects among factors and the chaotic effect on the target results, which will lead to the evaluation results of the final results more inclined to the dangerous state, and the fuzzy evaluation method cannot take into account the random effects among factors and the chaotic effect on the target results. If the structural maintenance and repair work based on this can make the structure in the "over-safe" state, the structural foundation load will increase, which will also have a certain impact on the operation of the structure, but also lead to a certain waste of manpower, material resources and financial resources.

Therefore, in this paper, the multi-level fuzzy evaluation method based on cloud theory was used to evaluate the safety level of the overall structure of the aqueduct, and the ambiguity and uncertainty of the multifactor coupling effect affecting the structural safety were considered. The fuzzy interaction process between the five aspects of structural form, seismic performance, bearing capacity, service status and durability defect was quantitatively described and judged. The uncertainty characteristics such as fuzzy randomness between various factors were integrated to ensure the comprehensiveness and scientificity of the evaluation process. At the same time, the improved multi-level fuzzy evaluation method of cloud theory guarantees the systematic and conciseness of the evaluation process, and couples the fuzzy randomness between factors. Besides, the expert's experience and knowledge were processed in an informational way. The entire analysis process can still be completed in the case of less quantitative information. The decision-making basis was more easily accepted and has high credibility and wide application scope, and has obvious advantages in solving unstructured decision- making problems. 


\section{CONCLUSION}

a) In order to evaluate the structural safety level, the problem of randomness and volatility of the influencing factors is less considered. The cloud theory is used to improve the multi-level fuzzy evaluation method. A scientific systematic analysis of the representative values of the initial state and service state of the aqueduct structure is carried out to ensure the reasonable display of various characteristics during the factorization process. The credibility of the evaluation method is improved, and the theoretical basis for the evaluation and repair measures of the structural service status is provided.

b) Through the fuzzy evaluation method improved by cloud theory, the comprehensive evaluation of the service state of the $5^{\#} \mathrm{U}$-type aqueduct structure in Jingdian Irrigation District was carried out, and the comprehensive cloud model parameters of the aqueduct were obtained as $\boldsymbol{W}=(82.3,76.2,79.8,78.4$, 75.1). After comparing with the standard cloud model, it is known that the security level of the structure has a tendency to develop to the III level, which was consistent with the actual results. The results of the cloud model showed that the overall safety level of the structure is good, and the defects of the main components are small, but the defects of the detailed components are large and there is the possibility of radiation damage to the main components. Therefore, it is necessary to pay attention to the maintenance and repair of the components.

c) The multi-level fuzzy evaluation method introduced by cloud theory has better reflected the ambiguity and volatility of the data itself, but there are still some shortcomings in the evaluation of the service status of the aqueduct: The weight cloud model and the membership degree cloud model of this paper were developed on the basis of the normal cloud model, but the normal cloud model cannot cover all system characteristics. In the subsequent structural evaluation, different cloud model types should be used for the overall evaluation of the structure to evaluate the results, and then acquire more accurate and reasonable results.

\section{FUTURE RESEARCH DIRECTIONS}

In further research, it is necessary to detect the forms of interaction of factors at all levels in the non-normal cloud state, and then improve the application scope of the cloud Theory. In this case, the fuzzy cloud theory can ensure covering all kinds of system characteristics with practical significance; hence it roundly improved the evaluation process and accurately evaluated the results.

In addition, other evaluation methods can also be considered in combination with the fuzzy cloud theory, thus integrating the advantages of various methods and combining the subjective and objective factors at all levels, and then systematically analyzing the confusion and uncertainty, macro and micro effects of all factors on the final results. Therefore, by using updated method to evaluate the safety of the structure, more reasonable maintenance methods can be adopted to extend the service and economic life of the target building.

Since the $5^{\#} \mathrm{U}$-type aqueduct is selected as the research object in this article, the basic structure and force mode of aqueducts are similar to bridges, tunnels and road sections. Therefore, the multistage fuzzy evaluation index system (Fig. 1) can be adjusted appropriately to better evaluate the similar buildings. Hence maintainers can use more economical measures for maintaining buildings and maximizing their life.

\section{Acknowledgments}

The authors are thankful for the State Key Research and Development Plan "Water Resource Efficient Development and Utilization" Key Special Project of China (Grant No. 2017YFC0405101); the National Natural Science Foundation of China (Grant No. 51279168); and the Open Fund of the State Key Laboratory of Permafrost Engineering (SKLFSE201801).

This work was supported by the National Natural Science Foundation of China (51579102/31360204), the Science and Technology Department of Henan Province (174200510020), the Henan University Science and Technology Innovation team support Program (19IRTSTHN030), the Ningxia Hui Autonomous region Water Science and Technology Project (TYZB-ZFCG2017-31).

\section{REFERENCES}

[1] Wang, J., Fei, L., Xie, F. et al. (2015). Comprehensive evaluation of operational status of large-scale irrigation district based on grey relation theory and TOPSIS method. Journal of Drainage and Irrigation Machinery Engineering, 33(11), 985-990.

[2] Volpi, E. \& Fiori, A. (2014). Hydraulic structures subject to bivariate hydrological loads: Return period, design, and risk assessment. Water Resources Research, 50(2), 885-897. https://doi.org/10.1002/2013WR014214

[3] Xu, C., Cheng, Y., Gao, Y. et al. (2018). Frost-resisting Durability of Concrete Structure Based on EAHP. Bulletin of the Chinese Ceramic Society, 37(04), 1181-1187.

[4] Sun, B. \& Xiao, R. (2015). Bridge Fire Risk Assessment System Based on Analytic Hierarchy Process-Fuzzy Comprehensive Evaluation. Journal of Tongji University (Natural Science), 43(11), 1619-1625.

[5] Zhan, J., Yan, Y., Zhang, N. et al. (2016). Study on dynamic evaluation method for substructures of railway simplysupported bridges. China Civil Engineering Journal, (10), 71-79.

[6] Fenocchi, A. \& Natale, L. (2016). Using Numerical and Physical Modeling to Evaluate Total Scour at Bridge Piers. Journal of Hydraulic Engineering, 142(3), 06015021. https://doi.org/10.1061/(ASCE)HY.1943-7900.0001096

[7] Hu, J., Zhong, J., Huang, S. et al. (2016). A method for bridge safety assessment based on reliability index. Journal of Harbin Engineering University, 37(4), 550-555.

[8] Mallick, J., Singh, R. K., Alawadh, M. A., et al. (2018). GISbased landslide susceptibility evaluation using fuzzy-AHP multi-criteria decision-making techniques in the Abha Watershed, Saudi Arabia. Environmental Earth Sciences, 77(7), 276. https://doi.org/10.1007/s12665-018-7451-1

[9] Singh, S., Olugu, E. U., Musa, S. N. et al. (2018). Fuzzybased sustainability evaluation method for manufacturing SMEs using balanced scorecard framework. Journal of Intelligent Manufacturing, 29(1), 1-18. https://doi.org/10.1007/s10845-015-1081-1 
[10] Parhoudeh, S., Baziar, A., Mazareie, A. et al. (2016). A novel stochastic framework based on fuzzy cloud theory for modeling uncertainty in the micro-grids. International Journal of Electrical Power \& Energy Systems, 80, 73-80. https://doi.org/10.1016/j.ijepes.2016.01.033

[11] He-Lin, F. U., Zhen, H., Huang, H. W. et al. (2017). Health diagnosis method of shield tunnel structure based on cloud theory. Chinese Journal of Engineering.

[12] Yang, Y. P. \& Shan, N. (2017). Evaluation of Shallow Groundwater Quality in Haikou Based on Fuzzy Comprehensive Evaluation Method. Ground Water.

[13] Xu, C., Cheng, H., Wang, Y. et al. (2017). Improved multilevel fuzzy evaluation model based on cloud theory for evaluation of soil salinization degree. Transactions of the Chinese Society of Agricultural Engineering, 33(24), 88-95.

[14] Tassa, A., Michele, S. D., Mugnai, A., et al. (2016). Cloud model-based Bayesian technique for precipitation profile retrieval from the tropical rainfall measuring mission microwave imager. Radio Science, 38(4), 39-1-39-13. https://doi.org/10.1029/2002RS002674

[15] Noh, Y., Hoffmann, F., \& Raasch, S. (2017). The Route to Raindrop Formation in a Shallow Cumulus Cloud Simulated by a Lagrangian Cloud Model. Journal of the Atmospheric Sciences, 74(7), 2125-2142. https://doi.org/10.1175/JAS-D-16-0220.1

[16] Tassa, A., Michele, S. D., Mugnai, A., et al. (2016). Cloud model-based Bayesian technique for precipitation profile retrieval from the Tropical Rainfall Measuring Mission Microwave Imager. Radio Science, 38(4) https://doi.org/10.1029/2002RS002674

[17] Han, X., An, X., \& Liu, C. (2018). Integrated risk evaluation of large river-crossing buildings in the Middle of the Southto-North Water Diversion Project. Journal of Tsinghua University (Science and Technology), 58(7), 639-649. https://doi.org/10.16511/j.cnki.ghdxxb.2018.25.030

[18] Xu, X. \& Yang, F. (2018). Risk assessment model of urban subsurface disease based on SPA and cloud theory. Advanced Engineering Sciences, 50(3), 224-232.

[19] Xu, X., Huang, Q., \& Ren, Y. (2017). Local variable weight and cloud theory applied in suspension bridges comprehensive assessment. Journal of Zhejiang University (Engineering Science), 51(8), 1544-1550.

[20] Wang, D., Liu, D., Ding, H., et al. (2016). A cloud modelbased approach for water quality assessment. Environmental Research, 149, 113. https://doi.org/10.1016/j.envres.2016.03.005

[21] Jiang, J., Han, G., Shu, L. et al. (2017).A Trust Model based on Cloud Theory in Underwater Acoustic Sensor Networks. IEEE Transactions on Industrial Informatics, PP(99), 1-1. https://doi.org/10.1109/TII.2015.2510226

[22] Wang, Y. (2018). Application of fuzzy comprehensive evaluation method in comprehensive evaluation of students' post practice and graduation design. Journal of Science of Teachers College \& University.

\section{Contact information:}

\section{Yu CHENG}

College of Water Resources and Architectural Engineering, Northwest A\&F University, Yangling 712100, Shaanxi, China

\section{Zhengzhong WANG}

(Corresponding author)

Institute of Water Engineering Safety Research in Arid and Cold Region,

Northwest A\&F University, Yangling 712100, Shaanxi, China

College of Water Resources and Architectural Engineering,

Northwest A\&F University, Yangling 712100, Shaanxi, China

Email: wangzz0910@163.com

\section{Cundong $\mathrm{XU}$}

School of Water Conservancy,

North China University of Water Resources and Electric Power,

Zhengzhou 450046, China

Henan Provincial Hydraulic Structure Safety Engineering Research Center,

Zhengzhou 450046, China

Hui CHENG

China Institute of Water Resources and Hydropower Research, Beijing 100038, China

\section{Xinglin ZHU}

School of Water Conservancy,

North China University of Water Resources and Electric Power

Zhengzhou 450046, China 